\title{
Effect of macrophage migration inhibitory factor (MIF) gene variants and MIF serum concentrations on the risk of type 2 diabetes: results from the MONICA/KORA Augsburg Case-Cohort Study, 1984-2002
}

\author{
C. Herder • N. Klopp • J. Baumert • M. Müller • \\ N. Khuseyinova • C. Meisinger • S. Martin • T. Illig • \\ W. Koenig • B. Thorand
}

Received: 6 June 2007 / Accepted: 19 July 2007 / Published online: 22 August 2007

(C) Springer-Verlag 2007

\begin{abstract}
Aims/hypothesis Macrophage migration inhibitory factor (MIF) is a central mediator of innate immunity. Our aim was to investigate the triangular association between MIF genotypes, circulating MIF concentrations and incident type 2 diabetes, and to use a Mendelian randomisation approach to assess the causal role of MIF.

Methods Using a case-cohort design within the population-based MONICA/KORA Augsburg Study, based on 502 individuals with incident type 2 diabetes $(293$ men, 209 women) and 1,632 non-cases (859 men, 773
\end{abstract}

Electronic supplementary material The online version of this article (doi:10.1007/s00125-007-0800-3) contains supplementary material, which is available to authorised users.

C. Herder $\cdot$ S. Martin

Institute for Clinical Diabetes Research, German Diabetes Centre, Leibniz Centre at Heinrich Heine University,

Dusseldorf, Germany

N. Klopp · J. Baumert $\cdot$ M. Müller ·

C. Meisinger $\cdot \mathrm{T}$. Illig $\cdot \mathrm{B}$. Thorand

GSF National Research Centre for Environment and Health, Institute of Epidemiology,

Neuherberg, Germany

\section{Müller}

LMU-IBE, Department of Epidemiology, University of Munich, Munich, Germany

N. Khuseyinova $\cdot$ W. Koenig $(\bowtie)$

Department of Internal Medicine II-Cardiology,

University of Ulm Medical Centre,

Robert-Koch-Str. 8,

89081 Ulm, Germany

e-mail: wolfgang.koenig@uniklinik-ulm.de women), we determined MIF serum levels at baseline and genotyped four MIF single nucleotide polymorphisms (SNPs).

Results The C allele of SNP rs1007888 (3.8 kb 3' of the translation termination codon) was associated with increased circulating MIF. MIF genotype rs $1007888 \mathrm{CC}$ was associated with an increased risk of type 2 diabetes in women [hazard ratio $(95 \%$ CI) $1.74(1.02-2.97)$ ], but not in men $[1.17$ (0.75-1.81)]. Elevated MIF serum levels were associated with higher type 2 diabetes risk also only in women [HR (95\% CI) 1.95 (1.15-3.29) comparing extreme quartiles after multiple adjustment], but not in men ( $p$ for interaction 0.039). The association between MIF levels and incident type 2 diabetes was significantly higher in obese women (111 cases, 147 non-cases) compared with nonobese women (98 cases, 626 non-cases; $p$ for BMI interaction 0.0002).

Conclusions/interpretation The consistent triangular relationship between genotypes, serum levels and incident type 2 diabetes in women indicates that MIF may play a causal role in the aetiology of type 2 diabetes and that elevated MIF levels confer a higher disease risk.

Keywords Cytokine $\cdot$ Inflammation $\cdot$ Macrophage migration inhibitory factor . Mendelian randomisation . Obesity · Single nucleotide polymorphism - Type 2 diabetes

$\begin{array}{ll}\text { Abbreviations } \\ \text { CRP } & \text { C-reactive protein } \\ \text { HR } & \text { hazard ratio } \\ \text { HWE } & \text { Hardy-Weinberg equilibrium } \\ \text { KORA } & \text { Kooperative Gesundheitsforschung in der }\end{array}$


Region Augsburg/Cooperative Health

Research in the Region of Augsburg

LD linkage disequilibrium

MI myocardial infarction

MIF macrophage migration inhibitory factor

MONICA Monitoring of Trends and Determinants in Cardiovascular Disease

S1/2/3 Survey $1 / 2 / 3$

SNP single nucleotide polymorphism

TC total cholesterol

\section{Introduction}

The link between macrophage migration inhibitory factor (MIF), a pleiotropic mediator of innate immunity $[1,2]$, and glucose metabolism has been addressed in molecular, cellular and clinical studies. Experiments with pancreatic islets and beta cell lines showed that high glucose concentrations augmented MIF expression, and that MIF stimulated insulin release [3, 4]. In addition, insulin increased MIF expression together with glucose [5]. This paracrine regulation suggested that elevated MIF levels might be part of a compensatory mechanism in early beta cell failure or insulin resistance to maintain sufficient insulin levels and thus indicate increased diabetes risk [6]. This hypothesis is in line with observations that systemic MIF concentrations are elevated in impaired glucose tolerance and type 2 diabetes [7, 8]. In morbidly obese women, high MIF levels were associated with decreased beta cell function, and individuals with high reductions in systemic MIF levels during a weight loss programme showed improved insulin resistance and beta cell function [9]. The only prospective data on MIF and diabetes risk come from the Finnish Diabetes Prevention Study. High MIF levels at baseline did not predict progression from impaired glucose tolerance to type 2 diabetes in the control group, but appeared to be associated with a better response to lifestyle intervention [10]. Further studies explored the role of MIF in obesity, which is also associated with chronic subclinical immune activation. MIF mRNA levels in peripheral blood mononuclear cells and serum levels are increased in obesity [11]; MIF protein is released by human adipocytes and its secretion is positively correlated with donor BMI [12].

At present, it is not clear whether elevated MIF levels represent a cause or the consequence of hyperglycaemia. In order to investigate the link between MIF and the risk of type 2 diabetes, a Mendelian randomisation approach can be utilised $[13,14]$. The association between elevated MIF levels and incident type 2 diabetes may be attributable to reverse causation or residual confounding. However, the additional analysis of the relationship between single nucleotide polymorphisms (SNPs) within the MIF gene and both MIF concentrations in the circulation and type 2 diabetes incidence could reveal whether MIF really plays a causal role in the development of type 2 diabetes. Genotypes that are associated with high MIF levels and are randomly transmitted to carriers from their parents may also be associated with higher disease risk for the carriers of these genotypes. Potentially confounding factors should be distributed evenly among those with high-risk or low-risk genotypes. In addition, genotypes are determined before the incidence of type 2 diabetes and are thus not subject to reverse causation.

There is evidence that there may indeed exist MIF genotypes that modulate MIF levels and thus enable such a Mendelian randomisation approach. The MIF gene on chromosome 22q11.2 consists of three short exons and two introns. An SNP $(-173 \mathrm{G}>\mathrm{C}$; rs755622) and a CATT tetranucleotide repeat $\left(-794 \mathrm{CATT}_{5-8}\right)$ in the promoter region have been tested for their associations with inflammatory diseases. Associations for one or both polymorphisms with systemic-onset juvenile idiopathic arthritis, rheumatoid arthritis, atopy, psoriasis, systemic lupus erythematosus and obesity have been reported [15-21] and suggest that these SNPs may have some functional relevance.

The aim of the present study was to investigate the triangular relationship between MIF levels, MIF gene variants and incident type 2 diabetes. We determined MIF genotypes for four SNPs in the promoter, in intron 2 and in the $3^{\prime}$-region, as well as circulating MIF levels in the large, prospective population-based Monitoring of Trends and Determinants in Cardiovascular Disease (MONICA)/Kooperative Gesundheitsforschung in der Region Augsburg [Cooperative Health Research in the Region of Augsburg (KORA)] case-cohort study to address the following questions: (1) do elevated MIF levels precede the onset of type 2 diabetes; (2) which are the determinants of circulating MIF levels: MIF genotypes or traditional (i.e. anthropometric, metabolic or lifestyle) risk factors; and (3) are MIF genotypes directly associated with type 2 diabetes risk.

\section{Methods}

Study population The design of this prospective casecohort study [22], conducted within the population-based MONICA/KORA cohort, has recently been described in detail [23-28]. For detailed information on the study population see the Electronic supplementary material (ESM).

The present study was based on 2,134 participants (293 men, 209 women with incident type 2 diabetes; 859 men, 773 women without incident type 2 diabetes). Mean follow- 
up time $( \pm \mathrm{SD})$ was $10.1 \pm 4.9$ years. For all analyses which included genetic markers the number of individuals was lower, since DNA samples were missing for about $10 \%$ of all individuals. For cross-sectional analyses, DNA samples were available in 1,872 participants $(1,001$ men, 871 women). For analyses with type 2 diabetes as end-point, DNA samples were available for 2,067 participants $(1,142$ men, 925 women). However, individuals with and without available DNA did not differ regarding distribution of risk factors.

MIF ELISA MIF serum concentrations were measured with the Quantikine ELISA kit (R\&D Systems, Wiesbaden, Germany). Intra- and inter-assay variations were determined using three controls with recombinant MIF and three sera in duplicates on 43 plates. Mean intra- and inter-assay CV values were 2.6 and $5.1 \%$, respectively, for the recombinant controls, and 3.8 and $11.1 \%$, respectively, for the sera.

SNPs, haplotypes and genotyping method In order to obtain complete coverage of the MIF gene, four SNPs were selected. SNPs rs2070766 (in intron 2: IVS2-6C >G), rs2070767 and rs1007888 (both in the 3'-flanking region) were selected according to HapMap information (http:// www.hapmap.org, last accessed in July 2007) in May 2005 for a $10 \mathrm{~kb}$ locus of the MIF gene. SNP rs 1007888 is 3,807 bp $3^{\prime}$ of the translation termination codon, but was included as it is a HapMap tagging SNP with a minor allele frequency $\mathrm{G}$ of 0.425 in persons with European descent (http://www.hapmap.org). SNP rs755622 in the promoter region $(-269 \mathrm{G}>\mathrm{C}$, also known as $-173 \mathrm{G}>\mathrm{C})$ was selected because associations of this SNP with inflammatory diseases have been reported before [15-21]. This SNP had not been genotyped by the HapMap project, and it was not known at the time of SNP selection that rs755622 is in almost complete linkage disequilibrium (LD) with rs2070766. The four SNPs that were selected covered the total common variation in the MIF gene at the time of SNP selection.

Genotyping was carried out with matrix-assisted laser desorption ionisation-time of flight analysis of alleledependent primer extension products as described [29]. Overall genotyping success rates were 99.2, 99.0, 99.4 and $99.3 \%$ for rs 755622 , rs 2070766 , rs2070767 and rs 1007888 , respectively.

Haplotype blocks were identified through examination of LD between consecutive SNPs in the subcohort. Consecutive SNPs with Lewontin's $D^{\prime} \geq 0.8$ were defined to lie in the same haplotype block. Haplotypes within these blocks were estimated using the estimation maximisation algorithm presented by Schaid et al. [30]. Due to the study design, haplotype estimation for analysis of incident type 2 diabetes had to be performed separately for cases and noncases. For association analysis within the subcohort, no distinction had to be made for haplotype estimation. The most frequent haplotype was chosen as reference. For all other haplotypes with an overall frequency of $\geq 1 \%$, separate variables indicating the individually expected number of copies of the haplotype were created. Less frequent haplotypes were pooled in a variable for rare haplotypes.

Statistical analyses Most aspects of the statistical analyses have been previously described (see ESM) [23-27]. For all SNPs, Hardy-Weinberg equilibrium (HWE) was tested using SAS procedure PROC ALLELE. Pairwise LD was measured using Lewontin's $D^{\prime}$ and the squared correlation coefficient $r^{2}$. Haplotype association analysis was carried out for the individually expected number of copies within each haplotype with frequency $\geq 1 \%$ (reference haplotype excluded) and the pooled group of rare haplotypes. This approach is closely related to the haplotype trend regression described by Zaykin et al. [31]. In contrast to Zaykin et al., we preferred to model expected numbers of copies instead of the haplotypes' probabilities.

In general, $p<0.05$ was considered statistically significant. In our analysis of the associations between the four MIF SNPs and incident type 2 diabetes, we calculated the number of effectively independent tests from the correlation structure between the four SNPs according to Li and Ji [32]. Given three effective SNPs, stratified analyses for men and women and two outcomes (serum levels and type 2 diabetes), the significance level adjusted for multiple testing according to Bonferroni for each of the SNPs, therefore, was 0.0042 for both the random subcohort and the type 2 diabetes case-cohort. All evaluations were performed with the statistical software package SAS (Version 8.02 for Unix, Version 9.1 for Windows; SAS Institute, Cary, NC, USA) and the statistical analysis software R, Version 2.2.1 [33].

\section{Results}

Study population Baseline demographic, clinical, immunological and lifestyle characteristics of the study participants have been described for almost identical samples [23, 25]. Data for the present sample are given in ESM Table 1. Briefly, individuals who developed type 2 diabetes during the follow-up period (cases) differed significantly from individuals without later onset of type 2 diabetes (noncases) by higher age, BMI, WHR, prevalence of hypertension, prevalence of myocardial infarction (MI), total cholesterol (TC):HDL-cholesterol ratio, C-reactive protein (CRP), IL-6 and frequency of smokers, and a lower level of 
Table 1 MIF serum concentrations $(\mathrm{ng} / \mathrm{ml})$ in the study population at baseline

${ }^{\mathrm{a}}$ Test for $\log _{e}$ MIF and adjusted for age, sex (in the whole sample only) and survey

\begin{tabular}{lllllll}
\hline & \multicolumn{2}{l}{ Type 2 diabetes cases } & & & Non-cases & \multicolumn{2}{c}{$p$ value $^{\text {a }}$} \\
\cline { 2 - 3 } & Median (25th-75th percentiles) & $n$ & & Median (25th-75th percentiles) & $n$ & \\
\hline All & $18.5(14.9-23.3)$ & 502 & & $17.7(14.1-22.3)$ & 1,632 & 0.042 \\
Men & $18.6(15.5-23.3)$ & 293 & & $18.4(15.1-23.9)$ & 859 & 0.79 \\
Women & $18.3(14.6-23.5)$ & 209 & $16.5(13.1-21.0)$ & 773 & 0.0035 \\
\hline
\end{tabular}

physical activity and of education at baseline compared with non-cases,

Systemic MIF concentrations and risk of type 2 diabetes In a cross-sectional analysis in the randomly sampled subcohort $(n=2,077)$, MIF serum levels (median [25th-75th percentiles]) were significantly higher in men than in women [18.7 ng/ml (15.3-24.0) vs $17.2 \mathrm{ng} / \mathrm{ml}(13.5-21.6) ; p<$ 0.0001]. The analysis of MIF baseline levels showed significantly higher levels for incident type 2 diabetes cases compared with non-cases (Table 1, $n=2,134$ ). The difference in MIF levels between type 2 diabetes cases and non-cases was highly significant only in women ( $p$ for interaction 0.009).

In Cox proportional hazards models, elevated MIF concentrations were significantly associated with higher type 2 diabetes risk in women, whereas the opposite trend was observed in men (Table 2). Adjustment for established risk factors of type 2 diabetes including BMI attenuated the hazard ratios (HRs) when comparing extreme MIF quartiles, but the sex difference persisted (model 3: $p$ for interaction 0.040). Addition of CRP and IL-6 to the model had no substantial effect on HRs (model 4: $p$ for interaction 0.039).

Model 3 was also calculated adjusting for WHR instead of BMI. WHR was only available for participants of Survey 2 (S2) and S3 (719 men, 602 women). We chose the same MIF quartiles as for the whole study population. In this subsample, the associations between MIF levels and incident type 2 diabetes were rather similar in men and women adjusting either for BMI [HR $(95 \% \mathrm{CI})$ for comparing extreme quartiles $0.49(0.27-0.89)$ in men, $2.55(1.23-5.30)$ in women] or for WHR [HR $(95 \% \mathrm{CI})$ for comparing extreme quartiles $0.56(0.31-1.00)$ in men, $2.37(1.17-4.81)$ in women].

Circulating MIF levels have been described to vary by circadian rhythm [34], and time of blood draw was also associated with MIF levels in the total subcohort of our study [median MIF levels $18.0 \mathrm{ng} / \mathrm{ml}$ before 11:00 hours $(n=522), 18.3 \mathrm{ng} / \mathrm{ml}$ from 11:00-13:59 hours $(n=562)$, $18.5 \mathrm{ng} / \mathrm{ml}$ from 14:00-16:59 hours $(n=642)$ and $16.8 \mathrm{ng} / \mathrm{ml}$ from 17:00 hours $(n=329)]$. When time of blood draw was added to model 3, HRs were virtually unchanged. MIF levels remained associated with type 2 diabetes risk in women [HR $(95 \% \mathrm{CI})$ for quartiles 2,3 and $4: 1.90$ (1.113.26), 1.64 (0.96-2.81) and 1.89 (1.11-3.22), respectively; $p$ for trend 0.039] and were not associated with type 2 diabetes risk in men [HR $(95 \% \mathrm{CI})$ for quartiles 2,3 and 4 : $0.92(0.60-1.41), 0.91(0.59-1.42)$ and $0.70(0.44-1.11)$, respectively; $p$ for trend 0.11 .

Post hoc subgroup analyses were performed stratified by BMI ( $<30 \mathrm{vs} \geq 30 \mathrm{~kg} / \mathrm{m}^{2}$ ). In non-obese women (98 cases, 626 non-cases), HRs $(95 \% \mathrm{CI})$ for MIF quartiles 2,3 and 4 were $1.21(0.65-2.25), 1.12(0.58-2.17)$ and $1.52(0.79$ 2.94), respectively, in model 3 . However, in obese women

Table 2 HRs (95\% CIs) of developing type 2 diabetes comparing quartiles of MIF serum concentrations

\begin{tabular}{|c|c|c|c|c|c|}
\hline Model & Quartile 1 & Quartile 2 & Quartile 3 & Quartile 4 & $p$ value (trend) \\
\hline \multicolumn{6}{|l|}{ Men } \\
\hline 1 & 1.0 & $0.97(0.67-1.42)$ & $1.05(0.72-1.53)$ & $1.02(0.70-1.48)$ & 0.84 \\
\hline 2 & 1.0 & $0.98(0.66-1.45)$ & $0.99(0.67-1.47)$ & $0.97(0.65-1.43)$ & 0.88 \\
\hline 3 & 1.0 & $0.88(0.58-1.35)$ & $0.85(0.55-1.30)$ & $0.67(0.42-1.05)$ & 0.08 \\
\hline 4 & 1.0 & $0.89(0.58-1.36)$ & $0.86(0.56-1.32)$ & $0.68(0.43-1.07)$ & 0.09 \\
\hline \multicolumn{6}{|l|}{ Women } \\
\hline 1 & 1.0 & $1.35(0.89-2.07)$ & $1.42(0.93-2.17)$ & $2.18(1.44-3.30)^{* * *}$ & 0.0003 \\
\hline 2 & 1.0 & $1.41(0.91-2.19)$ & $1.35(0.87-2.09)$ & $1.97(1.29-3.02)^{* *}$ & 0.0028 \\
\hline 3 & 1.0 & $1.75(1.04-2.96)^{*}$ & $1.60(0.96-2.69)$ & $1.83(1.10-3.05)^{*}$ & 0.034 \\
\hline 4 & 1.0 & $1.86(1.08-3.20)^{*}$ & $1.70(1.00-2.89)$ & $1.95(1.15-3.29)^{*}$ & 0.023 \\
\hline
\end{tabular}

Model 1: crude; model 2: adjusted for age and survey; model 3: adjusted for factors in model 2+BMI, systolic blood pressure, TC/HDLcholesterol ratio, physical activity, alcohol intake, smoking status, prevalent MI; model 4: adjusted for factors in model 3+CRP and IL-6 ${ }^{*} p<0.05$ compared with quartile 1

$* * p<0.01$ compared with quartile 1

$* * * p<0.001$ compared with quartile 1 
(111 cases, 147 cases), HRs were considerably higher and reached 3.00 (1.23-7.28), $2.97(1.22-7.24)$ and 2.54 (1.076.01) for MIF quartiles 2,3 and 4 . These data suggest that BMI is an effect modifier in the association between MIF and type 2 diabetes risk in women ( $p$ for BMI interaction 0.0002), whereas in men, the interaction with BMI appeared opposite, but was not significant [HR $(95 \% \mathrm{CI})$ for quartiles 2, 3 and 4 in non-obese men in model 3: 0.89 (0.51-1.52), $1.22(0.71-2.09)$ and 0.91 (0.53-1.57); HR $(95 \% \mathrm{CI})$ for quartiles 2,3 and 4 in obese men: $0.69(0.30$ $1.59), 0.46(0.21-1.05)$ and $0.38(0.15-0.91) ; p$ for BMI interaction 0.074].

Determinants of MIF concentrations in serum In order to characterise sex-specific determinants of MIF serum levels, which could confound the association between MIF levels and incident type 2 diabetes, the association of MIF levels with a range of anthropometric, clinical, immunological and lifestyle-related risk factors of type 2 diabetes was analysed in the subcohort separately for both sexes. In men, high MIF levels were significantly associated with smoking, high BMI, low HDL-cholesterol, high TC/HDL-cholesterol ratio and high CRP and IL-6 levels, whereas in women, high systolic blood pressure and high TC/HDL-cholesterol were significantly associated with high MIF levels (data not shown). Sex differences were significant for the associations of systemic MIF concentrations with BMI (higher positive correlation in men; $p=0.040)$ and $\log _{e} \operatorname{CRP}(p=0.048)$.

Association of SNPs and haplotypes with MIF serum levels We also investigated the impact of four SNPs on MIF serum levels in the subcohort. Genotype frequencies for the randomly sampled subcohort are shown in Table 3, and detailed genotype frequencies stratified for cases/non-cases and men/women are shown in ESM Table 2. No deviations from HWE were found $(p>0.05)$. High $D^{\prime}$ between neighbouring SNPs indicated that all SNPs lie in the same haplotype block, and haplotypes should be estimated over the complete set of SNPs (Fig. 1). In addition, rs755622 and rs2070766 were highly correlated $\left(r^{2}=0.955\right)$ and provided rather similar information. As shown in Table 4, MIF serum levels were higher in carriers of the $\mathrm{C}$ allele of rs 1007888 in men $\left(p_{\text {ANOVA }}=0.009, p_{\text {add }}=0.004\right)$ and, even more pronounced, in carriers of the T allele of rs 2070767 or the $\mathrm{C}$ allele of $\mathrm{rs} 1007888$ in women $\left(p_{\mathrm{ANOVA}}=0.005, p_{\mathrm{add}}=\right.$ 0.003 and $p_{\mathrm{ANOVA}}=3 \times 10^{-8}, p_{\text {add }}=2 \times 10^{-8}$, respectively). To assess whether the association of MIF SNPs with MIF serum levels was confounded by other factors including metabolic variables, we adjusted for the continuous variables age, BMI, systolic blood pressure, TC/HDLcholesterol, CRP, IL-6 and the categorical variables sex, survey, smoking status, alcohol consumption, physical activity, time of blood draw, prevalent MI and prevalent
Table 3 Frequencies of $M I F$ genotypes in the randomly sampled subcohort $(n=1,872)$

\begin{tabular}{llll}
\hline SNP & $\begin{array}{l}\text { Allele } \\
(1 / 2)\end{array}$ & $n(11 / 12 / 22)$ & Weighted frequency (\%) \\
\hline rs755622 & G/C & $1,317 / 488 / 44$ & $71.1 / 26.4 / 2.4$ \\
rs2070766 & $\mathrm{C} / \mathrm{G}$ & $1,315 / 485 / 45$ & $71.1 / 26.3 / 2.6$ \\
rs2070767 & $\mathrm{C} / \mathrm{T}$ & $1,075 / 684 / 94$ & $58.1 / 36.9 / 5.0$ \\
rs1007888 & $\mathrm{T} / \mathrm{C}$ & $555 / 954 / 342$ & $30.6 / 51.2 / 18.3$ \\
\hline
\end{tabular}

Genotype information was not available for all 1,872 participants, but genotyping success rates were $\geq 99.0 \%$ for each SNP

diabetes. The multiple adjustment revealed that the associations between MIF SNPs and MIF serum levels were not confounded by these variables (data not shown).

Further analysis investigating the associations of $M I F$ haplotypes and MIF serum levels confirmed the aforementioned results, as significant associations were only observed for the most common haplotypes designated by variants in loci identified through the analysis of single SNPs. MIF levels entered the model as $\log _{e}$ MIF, all results were adjusted for age and survey. MIF haplotypes and their frequencies are shown in Table 5. The most common haplotype, G-C-C-T (rs755622-rs2070766-rs2070767rs1007888), was set as reference. In men, only haplotype 2 $(\mathrm{G}-\mathrm{C}-\mathrm{T}-\mathrm{C})$ was associated with higher MIF levels $(\beta=0.056$; $p=0.009$ ), whereas in women the impact of rs2070767T and rs $1007888 \mathrm{C}$ was stronger and highly significant for both haplotype $2\left(\mathrm{G}-\mathrm{C}-\mathrm{T}-\mathrm{C} ; \beta=0.097 ; p=6 \times 10^{-5}\right)$ and haplotype $3\left(\mathrm{G}-\mathrm{C}-\mathrm{C}-\mathrm{C} ; \beta=0.090 ; p=8 \times 10^{-4}\right)$.

Genotypes, haplotypes and their association with incident type 2 diabetes In men, there were no significant associations between any of the SNPs and incident type 2 diabetes (Table 6). In contrast, women had a 1.7-fold increased risk ( $p=0.041)$ of developing type 2 diabetes if they were homozygous for the rs1007888C allele (Table 6), which was also associated with the highest MIF serum levels (Table 4). However, none of the genotype-sex interactions was significant (all $p>0.2$ ). Additive models revealed similar results to models described above. In women, one copy of the rs $1007888 \mathrm{C}$ allele was associated with an HR of $1.32(95 \%$ CI $1.01-1.72, p=0.042)$. All other associations in the additive model were not significant. The association between rs $1007888 \mathrm{C}$ and type 2 diabetes risk appeared slightly stronger in obese women compared with non-obese women (data not shown), but a formal test for interaction did not reach significance $(p=0.46)$.

Finally, MIF haplotypes were investigated with respect to their association with incident type 2 diabetes (adjusted for age and survey). None of the more common haplotypes (frequency $>5 \%$ ) was predictive of type 2 diabetes. 
Fig. 1 Analysis of LD of the four SNPs within the $M I F$ gene presented along with their physical distance on chromosome 22

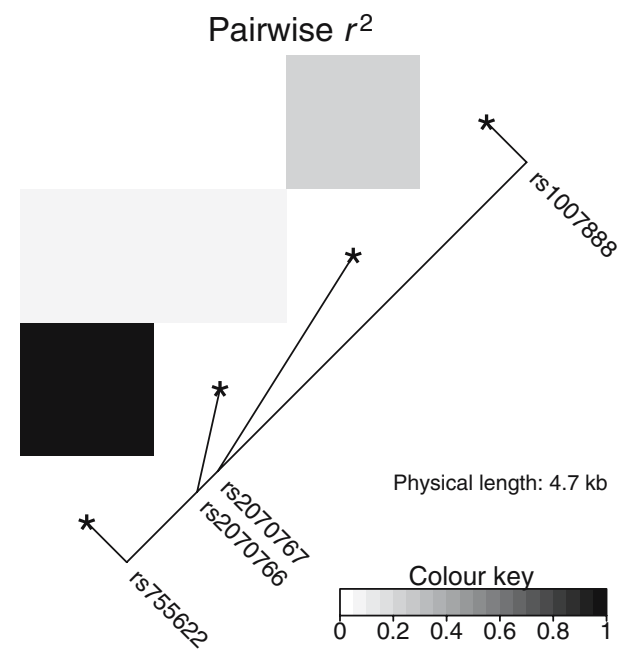

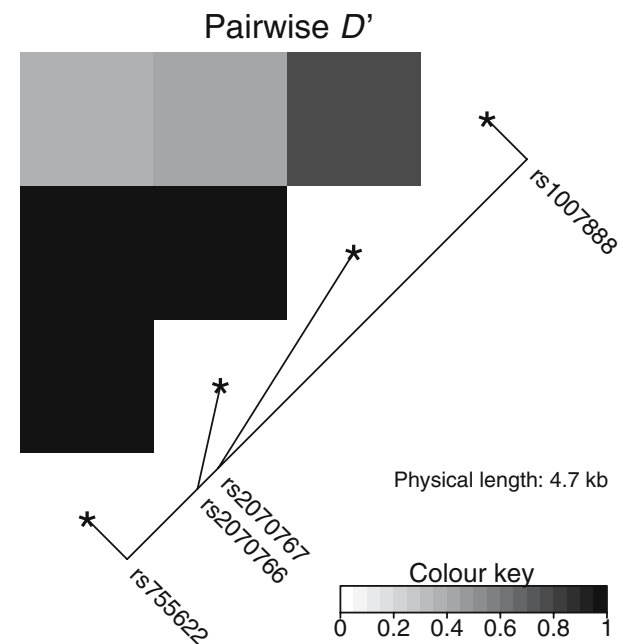

However, haplotype 5 was associated with increased type 2 diabetes risk in men [estimated frequencies in cases and non-cases 5.1 and 3.4\%, respectively; HR (95\% CI) 1.72 $(1.07-2.77) ; p=0.027]$. In the fully adjusted model (adjusted for age, survey, BMI, smoking, frequency of exercise, alcohol consumption, systolic blood pressure and TC/HDLcholesterol), the HR was increased further [HR 2.41 (1.473.97); $p=0.0005]$.

\section{Discussion}

The present study extends previous investigations in several important aspects. First, it demonstrates an association between elevated MIF levels and incident type 2 diabetes in women from a large population-based prospective cohort, whereas for men, the opposite trend was observed.

Second, it includes MIF genotypes and their association with circulating MIF levels and type 2 diabetes and thus allows utilisation of a Mendelian randomisation approach to investigate the link between MIF and the risk of type 2 diabetes. The $\mathrm{C}$ allele of rs 1007888 , an SNP that lies $3.8 \mathrm{~kb} 3^{\prime}$ of the translation termination codon and is associated with higher circulating MIF levels, was associated with a 1.7-fold increased risk of incident type 2 diabetes in women. Although the mechanism by which the $\mathrm{C}$ allele of rs1007888 increases circulating MIF levels is not known and our positive finding regarding the association between rs1007888 and incident type 2 diabetes was not significant after adjustment for multiple testing, our data still suggest that MIF might play a causal role in diabetes development rather than being a mere marker of beta cell failure or hyperinsulinaemia. In other words, this study provides evidence that elevated MIF levels are a cause and not a consequence of type 2 diabetes in women.

Interestingly, we did not observe any significant association between rs755622 $(-173 \mathrm{G}>\mathrm{C})$ and serum levels or incident type 2 diabetes. Previous studies indicated that this SNP may modulate $M I F$ expression in vitro [15, 21], affect circulating MIF levels in vivo [15, 21, 35] and increase the susceptibility to several inflammatory diseases [15-21]. However, opposite effects of rs755622 genotypes on gene expression were observed in different cell types $[15,21]$

Table 4 Associations of MIF genotypes and MIF serum concentrations (adjusted for age and survey) in the randomly sampled subcohort ( $n=1,872$ )

\begin{tabular}{|c|c|c|c|c|c|c|}
\hline \multirow[t]{2}{*}{ SNP } & \multirow[t]{2}{*}{ Sex } & \multicolumn{3}{|c|}{ Mean (SE) for genotypes } & \multirow[t]{2}{*}{$p$ value (ANOVA) } & \multirow[t]{2}{*}{$p$ value (additive) } \\
\hline & & 11 & 12 & 22 & & \\
\hline \multirow[t]{2}{*}{ rs755622 } & Men & $19.59(1.01)$ & $18.58(1.02)$ & $19.86(1.07)$ & 0.110 & 0.084 \\
\hline & Women & $17.41(1.02)$ & $17.07(1.02)$ & $15.83(1.06)$ & 0.260 & 0.183 \\
\hline \multirow[t]{2}{*}{ rs2070766 } & Men & $19.51(1.01)$ & $18.66(1.02)$ & $19.55(1.07)$ & 0.225 & 0.140 \\
\hline & Women & $17.45(1.02)$ & $16.99(1.02)$ & $15.68(1.06)$ & 0.156 & 0.102 \\
\hline \multirow[t]{2}{*}{ rs2070767 } & Men & $18.89(1.02)$ & $19.84(1.02)$ & $20.08(1.05)$ & 0.090 & 0.032 \\
\hline & Women & $16.68(1.02)$ & $18.18(1.02)$ & $17.77(1.05)$ & 0.005 & 0.003 \\
\hline \multirow[t]{2}{*}{ rs1007888 } & Men & $18.64(1.02)$ & $19.24(1.02)$ & $20.73(1.03)$ & 0.009 & 0.004 \\
\hline & Women & $16.10(1.02)$ & $17.15(1.02)$ & $19.71(1.03)$ & $3 \times 10^{-8}$ & $2 \times 10^{-8}$ \\
\hline
\end{tabular}

See Table 3 for allele codes $1 / 2$ 
Table 5 MIF haplotype frequencies in the randomly sampled subcohort ( $n=1,828: 974$ men, 854 women)

\begin{tabular}{llll}
\hline Number & Haplotype $^{\mathrm{a}}$ & \multicolumn{2}{l}{ Frequency (\%) } \\
\cline { 3 - 4 } & & Men & Women \\
\hline \multirow{2}{*}{ (ref) } & G-C-C-T & 41.3 & 41.0 \\
2 & G-C-T-C & 20.6 & 21.3 \\
3 & G-C-C-C & 19.9 & 19.1 \\
4 & C-G-C-T & 11.4 & 11.5 \\
5 & C-G-C-C & 3.7 & 3.7 \\
6 & G-C-T-T & 2.5 & 2.8 \\
Rare $^{\text {b }}$ & Sum (all other) & 0.6 & 0.6 \\
\hline
\end{tabular}

${ }^{a}$ Haplotypes are based on the SNPs rs755622-rs2070766-rs2070767rs 1007888

b The category 'rare haplotypes' contains all haplotypes with a frequency of $<1 \%$ in the subcohort

and the $\mathrm{C}$ allele was associated with significantly higher MIF levels in patients with juvenile idiopathic arthritis [21, 35], but not in patients with Crohn's disease [36], so that the functional impact of rs 755622 is not entirely clear. The absence of an association between this SNP and MIF serum levels in our population-based study may differ from observations in patient samples [21,35], but nevertheless is consistent with the lack of an association with incident type 2 diabetes.

Third, this study has sufficient power to look at sex differences. Based on the method developed by Cai and Zeng [37] for case-cohort studies, the power to detect significant sex differences in the association of an MIF SNP or MIF serum levels with incident type 2 diabetes assuming an HR modification of $50 \%$ was over $80 \%$ for each SNP and $98 \%$ for serum levels. So far, data on sex differences regarding MIF serum levels have been inconsistent $[7,38]$. This study indicates for the first time that MIF SNPs in the 3'-flanking region (rs2070767, rs1007888) are more strongly associated with circulating MIF protein levels in women than in men and that there are significant sex differences in the association of MIF genotypes and serum levels with incident type 2 diabetes as described above. The reason for this is unknown, but it can be speculated that MIF transcription or transcript stability may be influenced by sex hormones. Oestradiol stabilises oestrogen receptor RNA via sequences in the 3 '-untranslated region of the gene [39]. An effect of sex hormones is underlined by the finding that oestrogen regulates MIF production in murine and human monocytes and macrophages [40] via a nuclear factor $\mathrm{kB}$-dependent mechanism [41]. The fact that our study revealed not only an interaction with sex, but also with obesity, indicates that differences in fat distribution between men and women, and in particular in obese individuals, could also contribute to this phenomenon. Obese men tend to have more visceral fat than obese women, and women tend to have more subcutaneous fat than men, irrespectively of BMI. Data from the MONICA/ KORA study clearly demonstrate that for a given BMI, hip circumference is similar in men and women, whereas WHR is substantially higher in men than in women [42]. It is also known that sex differences exist in the associations between body composition, markers of inflammation and risk of type 2 diabetes [43, 44]. However, on the basis of data from this study it is not possible to explain why high MIF levels appear as significant risk factors in particular in obese women and are associated with reduced diabetes risk in
Table 6 HRs (95\% CIs) of developing type 2 diabetes comparing $M I F$ genotypes in 2,067 participants $(1,142$ men, 925 women)

\footnotetext{
${ }^{a}$ Model 1: adjusted for age and survey; model 2: adjusted for age, survey, BMI, smoking, alcohol, physical activity, systolic blood pressure, TC/HDLcholesterol, history of MI

${ }^{\mathrm{b}}$ See Table 3 for allele codes $1 / 2$ ${ }^{*} p=0.031$ compared with reference genotype 11

${ }^{* *} p=0.041$ compared with reference genotype 11
}

\begin{tabular}{|c|c|c|c|c|c|}
\hline \multirow[t]{2}{*}{ Sex } & \multirow[t]{2}{*}{ SNP } & \multirow[t]{2}{*}{ Model $^{\mathrm{a}}$} & \multicolumn{3}{|c|}{ Genotype $^{\mathrm{b}}$} \\
\hline & & & 11 & 12 & 22 \\
\hline \multirow[t]{8}{*}{ Men } & \multirow[t]{2}{*}{ rs 755622} & 1 & 1 (ref) & $1.07(0.80-1.44)$ & $0.82(0.36-1.84)$ \\
\hline & & 2 & 1 (ref) & $1.14(0.82-1.60)$ & $1.09(0.46-2.59)$ \\
\hline & \multirow[t]{2}{*}{ rs2070766 } & 1 & 1 (ref) & $1.06(0.79-1.43)$ & $1.11(0.51-2.40)$ \\
\hline & & 2 & 1 (ref) & $1.14(0.82-1.60)$ & $1.36(0.62-3.00)$ \\
\hline & \multirow[t]{2}{*}{ rs2070767 } & 1 & 1 (ref) & $1.09(0.83-1.44)$ & $1.11(0.57-2.16)$ \\
\hline & & 2 & 1 (ref) & $1.19(0.87-1.62)$ & $1.31(0.63-2.72)$ \\
\hline & \multirow[t]{2}{*}{ rs1007888 } & 1 & 1 (ref) & $1.01(0.74-1.37)$ & $0.99(0.67-1.47)$ \\
\hline & & 2 & 1 (ref) & $1.06(0.75-1.49)$ & $1.17(0.75-1.81)$ \\
\hline \multirow{8}{*}{ Women } & \multirow[t]{2}{*}{ rs 755622} & 1 & 1 (ref) & $0.94(0.64-1.38)$ & $1.57(0.69-3.61)$ \\
\hline & & 2 & 1 (ref) & $0.98(0.64-1.52)$ & $2.48(0.97-6.38)$ \\
\hline & \multirow[t]{2}{*}{ rs2070766 } & 1 & 1 (ref) & $0.93(0.64-1.36)$ & $1.55(0.68-3.56)$ \\
\hline & & 2 & 1 (ref) & $1.00(0.65-1.54)$ & $2.40(0.93-6.20)$ \\
\hline & \multirow[t]{2}{*}{ rs2070767 } & 1 & 1 (ref) & $1.18(0.84-1.65)$ & $0.93(0.42-2.04)$ \\
\hline & & 2 & 1 (ref) & $1.22(0.83-1.81)$ & $1.04(0.40-2.69)$ \\
\hline & \multirow[t]{2}{*}{ rs 1007888} & 1 & 1 (ref) & $1.05(0.72-1.54)$ & $1.65(1.05-2.61)^{*}$ \\
\hline & & 2 & 1 (ref) & $1.30(0.84-2.04)$ & $1.74(1.02-2.97)^{* *}$ \\
\hline
\end{tabular}


obese men, whereas associations with disease risk were much less pronounced in non-obese study participants.

Further investigations are required, but analysing the physiological role of MIF in the context of obesity and type 2 diabetes is complicated by two aspects of its biology. First, MIF is almost ubiquitously expressed [1], and cell-type specific differences in the regulation of MIF gene expression and production of the protein have been reported [45]. Second, many molecular mechanisms could explain why elevated MIF levels may contribute to the development of diabetes. MIF has been described as a proinflammatory cytokine and as potentiating lipopolysaccharide-mediated immune activation [46]. MIF counter-regulates glucocorticoid-mediated immune suppression, suppresses p53 activity, has enzyme activities and may also participate in antigen presentation $[1,46,47]$. However, no complete signal transduction pathway is known [48].

Major limitations of our study are the facts that more detailed information on body composition and fat depots as well as circulating sex hormone levels were not available. Strengths of the study include the use of the MONICA/ KORA cohort with a large number of cases and non-cases, long follow-up, and detailed information on immune markers and disease risk. In addition, the availability of both MIF genotypes and circulating MIF levels were important as they enabled a Mendelian randomisation approach to explore the potential causal link between MIF and type 2 diabetes.

Taken together, we investigated the triangular association between MIF levels, MIF genotypes and the risk of type 2 diabetes in a population-based case-cohort study. We found that elevated MIF serum levels are associated with increased risk of type 2 diabetes in women, but not in men. This association was stronger in obese compared with non-obese women. Serum MIF levels were significantly associated with MIF genotypes in women. Women who were homozygous for the rs1007888C allele, which was associated with the highest MIF serum levels, had a 1.7fold increased risk of type 2 diabetes. This combination of genetic and observational epidemiology points towards a causal role of MIF in the development of type 2 diabetes in women. The reason for the observed sex difference and for the impact of obesity is not known, so further research is required to characterise the mechanisms that link MIF and the risk of type 2 diabetes.

\footnotetext{
Acknowledgements The MONICA/KORA Augsburg cohort study was financed by the GSF National Research Center for Environment and Health and supported by grants from the Federal Ministry of Education and Research. The present study was funded by the German Research Foundation (DFG, TH-784/2-1), the German Federal Ministry of Education, Science, Research and Technology (National Genome Research Net-2, cardiovascular, 01GS0423), the German Federal Ministry of Health and Social Security and the Ministry of
}

Science and Research of the state North Rhine-Westphalia. We thank all members of the GSF Institute of Epidemiology who were involved in the planning and conduct of the MONICA/KORA Augsburg studies and the MONICA Augsburg survey team. We are grateful to L. Chambless (School of Public Health, University of North Carolina at Chapel Hill, NC, USA) for statistical advice concerning the analysis of the case-cohort dataset, to M. Kolz (GSF) for advice regarding the genotyping, to U. Poschen (German Diabetes Centre) for excellent technical assistance and to W. Rathmann (German Diabetes Centre) for critical reading of the manuscript. Finally, we are indebted to all study participants.

Duality of interest The authors declare that there is no duality of interest associated with this manuscript.

\section{References}

1. Calandra T, Roger T (2003) Macrophage migration inhibitory factor: a regulator of innate immunity. Nat Rev Immunol 3:791-800

2. Baugh JA, Donnelly SC (2003) Macrophage migration inhibitory factor: a neuroendocrine modulator of chronic inflammation. J Endocrinol 179:15-23

3. Waeber G, Calandra T, Roduit R et al (1997) Insulin secretion is regulated by the glucose-dependent production of islet beta cell macrophage migration inhibitory factor. Proc Natl Acad Sci U S A 94:4782-4787

4. Plaisance V, Thompson N, Niederhauser G et al (2002) The mif gene is transcriptionally regulated by glucose in insulin-secreting cells. Biochem Biophys Res Commun 295:174-181

5. Sakaue S, Nishihira J, Hirokawa J et al (1999) Regulation of macrophage migration inhibitory factor (MIF) expression by glucose and insulin in adipocytes in vitro. Mol Med 5:361-371

6. Waeber G, Calandra T, Bonny C, Bucala R (1999) A role for the endocrine and pro-inflammatory mediator MIF in the control of insulin secretion during stress. Diabetes Metab Res Rev 15:47-54

7. Herder C, Kolb H, Koenig W et al (2006) Association of systemic concentrations of macrophage migration inhibitory factor with impaired glucose tolerance and type 2 diabetes: results from the Cooperative Health Research in the Region of Augsburg, Survey 4 (KORA S4). Diabetes Care 29:368-371

8. Yabunaka N, Nishihira J, Mizue Y et al (2000) Macrophage migration inhibitory factor in patients with type 2 diabetes. Diabetes Care 23:256-258

9. Church TS, Willis MS, Priest EL et al (2005) Obesity, macrophage migration inhibitory factor, and weight loss. Int $\mathbf{J}$ Obes (Lond) 29:675-681

10. Herder C, Peltonen M, Koenig W et al (2006) Systemic immune mediators and lifestyle changes in the prevention of type 2 diabetes: results from the Finnish Diabetes Prevention Study. Diabetes 55:2340-2346

11. Ghanim H, Aljada A, Hofmeyer D, Syed T, Mohanty P, Dandona P (2004) Circulating mononuclear cells in the obese are in a proinflammatory state. Circulation 110:1564-1571

12. Skurk T, Herder C, Kräft I, Müller-Scholze S, Hauner H, Kolb H (2005) Production and release of macrophage migration inhibitory factor from human adipocytes. Endocrinology 146:1006-1011

13. Davey Smith G, Ebrahim S (2003) Mendelian randomization: can genetic epidemiology contribute to understanding environmental determinants of disease? Int J Epidemiol 32:1-22

14. Hingorani A, Humphries S (2005) Nature's randomised trials. Lancet 366:1906-1908

15. Donn R, Alourfi Z, De Benedetti F et al (2002) Mutation screening of the macrophage migration inhibitory factor gene. Positive association of a functional polymorphism of macrophage 
migration inhibitory factor with juvenile idiopathic arthritis. Arthritis Rheum 46:2402-2409

16. Barton A, Lamb R, Symmons D et al (2003) Macrophage migration inhibitory factor (MIF) gene polymorphism is associated with susceptibility to but not severity of inflammatory polyarthritis. Genes Immun 4:487-491

17. Hizawa N, Yamaguchi E, Takahashi D, Nishihira J, Nishimura M (2004) Functional polymorphisms in the promoter region of macrophage migration inhibitory factor and atopy. Am J Respir Crit Care Med 169:1014-1018

18. Donn RP, Plant D, Jury F et al (2004) Macrophage migration inhibitory factor gene polymorphism is associated with psoriasis. J Invest Dermatol 123:484-487

19. Sanchez E, Gomez LM, Lopez-Nevot MA et al (2006) Evidence of association of macrophage migration inhibitory factor gene polymorphisms with systemic lupus erythematosus. Genes Immun 7:433-436

20. Sakaue S, Ishimaru S, Hizawa N et al (2006) Promoter polymorphism in the macrophage migration inhibitory factor gene is associated with obesity. Int J Obes (Lond) 30:238-242

21. Donn R, Alourfi Z, Zeggini E et al (2004) A functional promoter haplotype of macrophage migration inhibitory factor is linked and associated with juvenile idiopathic arthritis. Arthritis Rheum 50:1604-1610

22. Barlow WE (1994) Robust variance estimation for the case-cohort design. Biometrics 50:1064-1072

23. Thorand B, Kolb H, Baumert J et al (2005) Elevated levels of interleukin-18 predict the development of type 2 diabetes: results from the MONICA/KORA Augsburg Study, 1984-2002. Diabetes 54:2932-2938

24. Thorand B, Baumert J, Chambless L et al (2006) Elevated markers of endothelial dysfunction predict type 2 diabetes mellitus in middle-aged men and women from the general population. Arterioscler Thromb Vasc Biol 26:398-405

25. Herder C, Baumert J, Thorand B et al (2006) Chemokines as risk factors for type 2 diabetes: results from the MONICA/KORA Augsburg Study, 1984-2002. Diabetologia 49:921-929

26. Herder C, Baumert J, Thorand B et al (2006) Chemokines and incident coronary heart disease: results from the MONICA/KORA Augsburg case-cohort study, 1984-2002. Arterioscler Thromb Vasc Biol 26:2147-2152

27. Koenig W, Khuseyinova N, Baumert J et al (2006) Increased concentrations of C-reactive protein and IL- 6 but not IL-18 are independently associated with incident coronary events in middleaged men and women: results from the MONICA/KORA Augsburg case-cohort study, 1984-2002. Arterioscler Thromb Vasc Biol 26:2745-2751

28. Thorand B, Löwel H, Schneider A et al (2003) C-reactive protein as a predictor for incident diabetes mellitus among middle-aged men: results from the MONICA Augsburg cohort study, 19841998. Arch Intern Med 163:93-99

29. Weidinger S, Klopp N, Wagenpfeil S et al (2004) Association of a STAT 6 haplotype with elevated serum IgE levels in a population based cohort of white adults. J Med Genet 41:658-663

30. Schaid DJ, Rowland CM, Tines DE, Jacobson RM, Poland GA (2002) Score tests for association between traits and haplotypes when linkage phase is ambiguous. Am J Hum Genet 70:425-434

31. Zaykin DV, Westfall PH, Young SS et al (2002) Testing association of statistically inferred haplotypes with discrete and continuous traits in samples of unrelated individuals. Hum Hered 53:79-91
32. Li J, Ji L (2005) Adjusting multiple testing in multilocus analyses using the eigenvalues of a correlation matrix. Heredity 95:221-227

33. R Development Core Team (2005) R: A language and environment for statistical computing. Vienna, Austria, R Foundation for Statistical Computing, 2005 (www.R-project.org)

34. Petrovsky N, Socha L, Silva D, Grossman AB, Metz C, Bucala R (2003) Macrophage migration inhibitory factor exhibits a pronounced circadian rhythm relevant to its role as a glucocorticoid counter-regulator. Immunol Cell Biol 81:137-143

35. De Benedetti F, Meazza C, Vivarelli M et al (2003) Functional and prognostic relevance of the -173 polymorphism of the macrophage migration inhibitory factor gene in systemic-onset juvenile idiopathic arthritis. Arthritis Rheum 48:1398-1407

36. Dambacher J, Staudinger T, Seiderer J et al (2007) Macrophage migration inhibitory factor (MIF) $-173 \mathrm{G} / \mathrm{C}$ promoter polymorphism influences upper gastrointestinal tract involvement and disease activity in patients with Crohn's disease. Inflamm Bowel Dis 13:71-82

37. Cai J, Zeng D (2004) Sample size/power calculation for casecohort studies. Biometrics 60:1015-1024

38. Mizue Y, Nishihira J, Miyazaki T et al (2000) Quantitation of macrophage migration inhibitory factor (MIF) using the one-step sandwich enzyme immunosorbent assay: elevated serum MIF concentrations in patients with autoimmune diseases and identification of MIF in erythrocytes. Int J Mol Med 5:397-403

39. Mitchell DC, Ing NH (2003) Estradiol stabilizes estrogen receptor messenger ribonucleic acid in sheep endometrium via discrete sequence elements in its 3 -untranslated region. Mol Endocrinol 17:562-574

40. Ashcroft GS, Mills SJ, Lei K et al (2003) Estrogen modulates cutaneous wound healing by downregulating macrophage migration inhibitory factor. J Clin Invest 111:1309-1318

41. Hardman MJ, Waite A, Zeef L, Burow M, Nakayama T, Ashcroft GS (2005) Macrophage migration inhibitory factor: a central regulator of wound healing. Am J Pathol 167:1561-1574

42. Meisinger C, Döring A, Thorand B, Heier M, Löwel H (2006) Body fat distribution and risk of type 2 diabetes in the general population: are there differences between men and women? The MONICA/KORA Augsburg cohort study. Am J Clin Nutr $84: 483-489$

43. Thorand B, Baumert J, Döring A et al (2006) Sex differences in the relation of body composition to markers of inflammation. Atherosclerosis 184:216-224

44. Thorand B, Baumert J, Kolb H et al (2007) Sex differences in the prediction of type 2 diabetes by inflammatory markers: results from the MONICA/KORA Augsburg case-cohort study, 19842002. Diabetes Care 30:854-860

45. Alourfi Z, Donn RP, Stevens A, Berry A, McMaster A, Ray DW (2005) Glucocorticoids suppress macrophage migration inhibitory factor (MIF) expression in a cell-type-specific manner. J Mol Endocrinol 34:583-595

46. Kudrin A, Scott M, Chung CW et al (2006) Human macrophage migration inhibitory factor (MIF): a proven immunomodulatory cytokine? J Biol Chem 281:29641-29651

47. Potolicchio I, Santambrogio L, Strominger JL (2003) Molecular interaction and enzymatic activity of macrophage migration inhibitory factor with immunorelevant peptides. J Biol Chem 278:30889-30895

48. Morand EF, Leech M, Bernhagen J (2006) MIF: a new cytokine link between rheumatoid arthritis and atherosclerosis. Nat Rev Drug Discov 5:399-410 